\title{
Nonwovens Uniformity Spatial Characterization *
}

\author{
Jiří Militký ${ }^{1+}$ and Václav Klička ${ }^{2}$ \\ ${ }^{1}$ Dept of Textile Materials, Technical University of Liberec, 46117 Liberec, Czech Republic \\ ${ }^{2}$ Rieteras, Ústín. Orlicí, Czech Republic
}

( Received 4 October 2006, accepted 4 February 2007)

\begin{abstract}
The main aim of this contribution is comparison of method for evaluation of nonwovens surface uniformity based on the data in the form of rectangular arrays (quadrat method). These data can be obtained from digital images where the variation of mass is characterizes by the variation of grey level image. The evaluation of uniformity is based on the variation coefficient model, ANOVA model and spatial descriptors of irregularity
\end{abstract}

Keywords: spatial heterogeneity, quadrat method, surface uniformity, ANOVA, spatial descriptors

\section{Introduction}

The products from nonwovens are nowadays applicable in the fields requiring relative high mass uniformity or uniformity of basic physico - mechanical properties. There exist a lot of methods for description of planar anisotropy and other structural characteristics of nonwovens (see [5] and [6]).

The spatial variation of geometric and other properties is the main peculiarity of textile products. For the purpose of design, quality control and application in composites it is necessary to have tools for expressing this variability by suitable characteristics. Especially products from nonwovens are nowadays applicable in the fields requiring relative high mass uniformity or uniformity of basic physico - mechanical properties [3]. There exist a lot of methods for description of planar anisotropy and other structural characteristics of nonwovens (see [5]; [6]; [9]). Selected methods of continuous and discontinuous measurement of planar uniformity of nonwovens are described in the dissertation [9]. In parallel to the description of unevenness of linear textile structures by the length variation function, there can be constructed surface variation function for textile fabrics. The surface variation function can be easily used for description of unevenness or uniformity. Another possibility is to use some techniques based on the spatial pattern analysis as variance to mean ratio.

The main aim of this work is attempt to describe surface irregularity of nonwoven textile structure based on the so-called quadrat method, where characteristic of quadrat is mean value of grey level. Principle is to divide sample to the rectangular net of cells named quadrats. In these quadrats the mean optical transparency (grey level) is evaluated. Direction $x$ is equivalent to the machine direction (index i). In this direction are $\mathrm{N}$ quadrats. Direction $y$ is equivalent to the cross direction (index $\mathrm{j}$ ). In this direction are M quadrats.

For evaluation of uniformity the five kinds of methods are useful.

First one is based on the computation of variation coefficient in selected directions (machine and cross direction), and testing the significance of their differences [7].

Second one is based on the modelling of data arrays by the ANOVA (analysis of variance) type models and testing hypothesis about homogeneity in selected directions [10].

Third one is based on the analysis of random field. The moment characteristics of second order as spatial covariance and variogram are used for description of these fields. The fractal dimension characterizing random field complexity can be computed directly from variogram [4].

Fourth one is based on the global and local spatial variation indices of Geary and Moran type [1].

Fifth one is based on the utilization of multivariate kurtoisis of indicator random variables [8]

\footnotetext{
* This work was supported by the research project 1M4674788501 of Czech Ministry of Education.

+ Corresponding author. Tel.: +420-48-5353255; fax: +420-48-5353542.

E-mail address: jiri.militky@tul.cz.
} 
There exist a lot of other characteristics as spatial descriptors of irregularity [6] suitable for special situations (point patterns).

The aim of this work is comparison of some characteristics of uniformity on the example of spun bonded lightweight nonwoven lap.

\section{Irregularity characterization}

Irregularity characterization is classically based on the coefficient of variation CV or derived statistics. For characterization of lattice data array the models based on the ANOVA principle are often used. For detailed description of irregularity field the second order characteristics as function of distance separation vector can be used as well. These characteristics can be compared with ideal models of nonwoven structures. Some simple indices can be obtained from indicator random variable, which is simply threshold of original spatial variable.

\subsection{Spatial lattice processes}

Spatial data are investigated on the specific domain D. Usually D is a subset of two-dimensional space, but generally the $d$ dimensional domain can be used and then $D \subset \mathfrak{R}^{d}$. The vector $\mathbf{s}$ contains information on the data location. In two-dimensional space, $\mathbf{s}$ has 2 components $(\mathrm{x}, \mathrm{y})$ containing the coordinates. At locations $\mathbf{s}$, the values of some variable $\mathrm{z}(\mathbf{s})$ of interests (grey level, mass, density, thickness etc.) are obtained. The $\mathrm{z}(\mathbf{s})$ is a random variable at each location. The general spatial model has the form $\{z(\mathbf{s}): \mathbf{s} \in D\}$.

There exist three basic model types:

1: Geostatistical data. Here D is a continuous fixed subset of $\mathfrak{R}^{d} ; \mathrm{z}(\mathbf{s})$ is a random vector at location $\mathbf{s} \in D$.

2: Lattice data. Here D is a fixed but countable subset of $\mathfrak{R}^{d}$ such as a grid some representation with nodes; $\mathrm{z}(\mathbf{s})$ is a random vector at locations $\mathbf{s} \in D$.

3: Point Patterns. Here D is a random subset of $\mathfrak{R}^{d}$ and is called a point process; if $z(\mathbf{s})$ is a random vector at location $\mathbf{s} \in D$ then it is a marked spatial point process; if $z(\mathbf{s}) \equiv 1$ so that it is a degenerate random variable, then only $\mathrm{D}$ is random and it is called a spatial point process.

For the quadrat method is quantity $\mathrm{z}(\mathbf{s})$ random function of two variables called random field. This random field is fully described by the $\mathrm{n}$ variate probability density function

$$
p_{n}\left(z_{1}, z_{2}, . . z_{n}\right)=P\left\{z_{i} \leq z\left(s_{\mathbf{i}}\right) \leq z_{i}+d z_{i}, \quad i=1 \ldots n\right\}
$$

Homogeneous random field has property of invariance according to the translation. The mean value $\mathrm{E}(\mathrm{z})$ is defined as

$$
E(z)=\int z p_{1}(z) d z
$$

Variability of random field is characterized by the covariance function

$$
C\left(\mathbf{s}_{1}, \mathbf{s}_{2}\right)=E\left(\left(z_{1}-E\left(z_{1}\right)\left(z_{2}-E\left(z_{2}\right)\right) .\right.\right.
$$

For the case when points $\mathbf{s}_{1}$ and $\mathbf{s}_{2}$ are coincident is covariance function reduced to the variance function $\mathrm{D}(\mathbf{s})$ defined as [10]

$$
D(\mathbf{s})=E\left(z(\mathbf{s})^{2}\right)-(E(z(\mathbf{s})))^{2} .
$$

Another measure of spatial variability is so called variogram or semivariogram defined as half of variance of the increment $\left(\mathrm{z}\left(\mathbf{s}_{1}\right)-\mathrm{z}\left(\mathbf{s}_{2}\right)\right)$

$$
\gamma\left(\mathbf{s}_{1}, \mathbf{s}_{2}\right)=0.5 * D\left[z\left(\mathbf{s}_{1}\right)-z\left(\mathbf{s}_{2}\right)\right]=\operatorname{Var}(z(\mathbf{u})-z(\mathbf{u}+\mathbf{h}))=\operatorname{Var}(z)(1-\rho(\mathbf{h}))
$$

For homogeneous random field is covariance function dependent on the distance between points

$\mathbf{s}_{1}=\left(\mathrm{x}_{1}, \mathrm{y}_{1}\right)$ and $\mathbf{s}_{2}=\left(\mathrm{x}_{2}, \mathrm{y}_{2}\right)$ only. For this case is $C\left(\mathbf{s}_{1}, \mathbf{s}_{2}\right)=C\left(x_{2}-x_{1}, y_{2}-y_{1}\right)$.

For isotropic random field is covariance function invariant against rotation and mirroring. This function is then dependent on the length $d=\sqrt{\left(x_{2}-x_{1}\right)^{2}+\left(y_{2}-y_{1}\right)^{2}}$ and therefore $C\left(\mathbf{s}_{1}, \mathbf{s}_{2}\right)=R(d)$. A random function $\mathrm{z}(\mathbf{s})$ is said to be second order stationary, if [2] 
- the mean value exists and is independent on the location vector $\mathrm{x}$, i.e. $\mathrm{E}(\mathrm{z})=\mathrm{m}$.

- for each pair of random variables $z(\mathbf{s})$ and $z(\mathbf{s}+\mathbf{h})$ is covariance dependent on the separation vector $\mathbf{h}$ only $C(\mathbf{h})=E[z(\mathbf{s}) * z(\mathbf{s}+\mathbf{h})]-m^{2}$

The stationarity of variance imply the stationarity of covariance and variogram

$$
D(z)=C(\mathbf{h}=0)=C(0) \quad \gamma(\mathbf{h})=C(0)-C(\mathbf{h}) .
$$

The second order stationarity implies that the covariance and variogram are the equivalent tools for characterization of spatial correlation. It is clear that second order stationarity leads to the continuity at origin because $\gamma(0)=0$.

If $\gamma(0)=c_{0}>0$, then $c_{0}$ is called as nugget effect (small scale variations cause discontinuity at origin). If $\gamma(\mathbf{h})=$ const. for all $\mathrm{h}$ then the $\mathrm{z}($.$) are uncorrelated in this direction.$

The dependence of $\gamma(\mathbf{h})$ on $\boldsymbol{h}$ can be expressed by the various parametrical models. Very often it is suitable to use the spherical model expressed in the form

$$
\gamma(h)=c_{0}+c\left[1.5(h / a)-0.5(h / a)^{3}\right] \text { for } 0 \leq h \leq a \text { or } \gamma(h)=c_{0}+c \text { for } h>a
$$

where $h$ is the length of $\boldsymbol{h}$. The distributional properties of variogram and techniques for parameter estimation are discussed in the book of [2].

For computation of sample estimators of above defined measures of spatial continuity the experimentally determined values of uniformity (grey level, planar densities or mass) $z\left(\mathbf{s}_{\mathrm{i}}\right)=\mathrm{z}(\mathrm{k}, \mathrm{j})$ of $\mathrm{k}, \mathrm{j}$ th cell $(\mathrm{k}=1 \ldots \mathrm{m}, \mathrm{j}$ $=1 \ldots \mathrm{n})$ of the rectangular net are used. The sample directional variogram function for chosen separation vector $\boldsymbol{h}$ is calculated according to the following formula

$$
\gamma(\mathbf{h})=\frac{1}{2 N(\mathbf{h})} \sum_{i=1}^{N(\mathbf{h})}\left[z\left(\mathbf{s}_{i}\right)-z\left(\mathbf{s}_{i}+\mathbf{h}\right)\right]^{2}
$$

where $\mathrm{N}(h)$ is number of points in separation distances $h$. For regularly distributed points $s$ are the separation distances multiples of distance between cells of net. Therefore it is possible to compute characteristics for directions $0^{\circ}\left(\boldsymbol{h}=\mathrm{c}^{*}[1,0]\right), 45^{\circ}\left(\boldsymbol{h}=\mathrm{c}^{*}[1,1]\right)$, and $90^{\circ}\left(\boldsymbol{h}=\mathrm{c}^{*}[1,0]\right)$ for lags $\mathrm{c}=1,2,3 \ldots$ only. Averaging of variograms calculated in all directions leads to the omnidirectional variogram. For computation of these spatial measures the program NONWP written in MATLAB 7.04 was created.

\subsection{Analysis based on $\mathrm{CV}$}

Surface uniformity is classically described by the coefficient of variation (CV). This coefficient is traditionally used as the characteristics of unevenness.

According to the common definitions we can simply computed the overall mean, variance and coefficient of variation

$$
m=\frac{1}{M N} \sum_{i} \sum_{j}\left(z_{i j}\right) \quad s^{2}=\frac{1}{M N} \sum_{i} \sum_{j}\left(z_{i j}-m\right)^{2} \quad C V=\frac{s}{m}
$$

Here $z_{\mathrm{ij}}$ is selected characteristic of quadrats (here mean grey level $m_{\mathrm{ij}}$ ). Direction $x$ is equivalent to the machine direction (index $i$ ). In this direction are $\mathrm{N}$ quadrats. Direction $y$ is equivalent to the cross direction (index $j$ ). In this direction are $\mathrm{M}$ quadrats.

The quantity $\mathrm{CV}$ is in fact external variation coefficient $\mathrm{CB}(F)$ between cell areas $F$.

Ideal value of $\mathrm{CV}$ for nonwoven of total weight $\mathrm{W}$ having Poisson distribution of random fibres of fineness TV and density $\rho_{V}$ is defined as [11]

$$
C V_{N}(P)=\frac{\sqrt[4]{\pi}}{\sqrt{2}} \sqrt[4]{\frac{T_{V} \rho_{V}}{W^{2}}}
$$

The total variance $\mathrm{s} 2$ can be divided to the two terms by using of means in the machine direction and cross direction

$$
m_{i o}=\frac{1}{M} \sum_{j} z_{i j} \quad m_{o j}=\frac{1}{N} \sum_{j} z_{i j}
$$

Symbol „, "“denotes index used for summation i.e. $m_{i o}$ is mean value for $i$ th position in the machine 
direction. For the machine direction (expansion of eqn.(14) by using of the $m_{\mathrm{io}}$ ) the following relation results [7]

$$
s^{2}=s_{L}^{2}+s_{H L}^{2}
$$

where the variance in the machine direction $s_{L}^{2}$ is

$$
s_{L}^{2}=\frac{1}{N} \sum_{i}\left(m_{i o}-m\right)^{2}
$$

and the variance in the transversal direction $s_{H L}^{2}$ is

$$
s_{H L}^{2}=\frac{1}{M N} \sum_{i} \sum_{j}\left(z_{i j}-m_{i o}\right)^{2}
$$

For the cross direction is

$$
s^{2}=s_{H}^{2}+s_{L H}^{2}
$$

where the variance in the cross-direction $s_{H}^{2}$ is

$$
s_{H}^{2}=\frac{1}{M} \sum_{j}\left(m_{o j}-m\right)^{2}
$$

and the variance in the longitudinal direction $s_{L H}^{2}$ is

$$
s_{L H}^{2}=\frac{1}{M N} \sum_{i} \sum_{j}\left(z_{i j}-m_{o j}\right)^{2}
$$

The coefficients of variation $\mathrm{CV}_{\mathrm{L}}, \mathrm{CV}_{\mathrm{HL}}, \mathrm{CV}_{\mathrm{H}}$ and $\mathrm{CV}_{\mathrm{LH}}$ are obtained by dividing the corresponding standard deviations by the mean value $m$. These coefficients are from statistical point of view the point estimates of population variation coefficients $\mathrm{CV}_{\mathrm{PL}}, \mathrm{CV}_{\mathrm{PH}}$, etc. For creation of confidence intervals the variance of point estimates have to be computed [10].

The uniformity of mass distribution can be also characterized by index of dispersion.

$$
I_{d}=\frac{s^{2}}{m}
$$

Spatial randomness corresponds to the Poisson distribution. The null hypothesis of randomness can be tested by comparison of Id with quantiles of $\chi^{2}$ distribution. It is possible to compute the limit ML bellow the pattern is uniform and limit MU above the pattern is clumped [6].

\section{Experimental part}

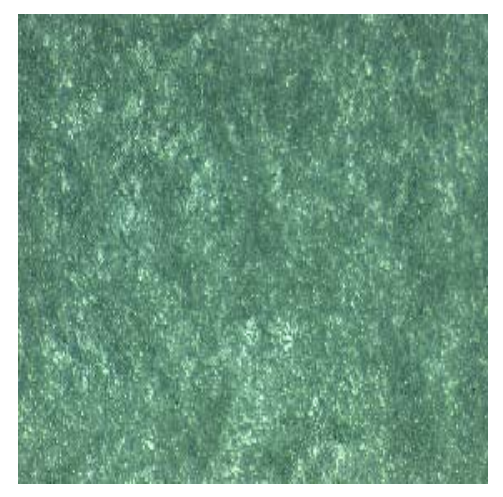

a

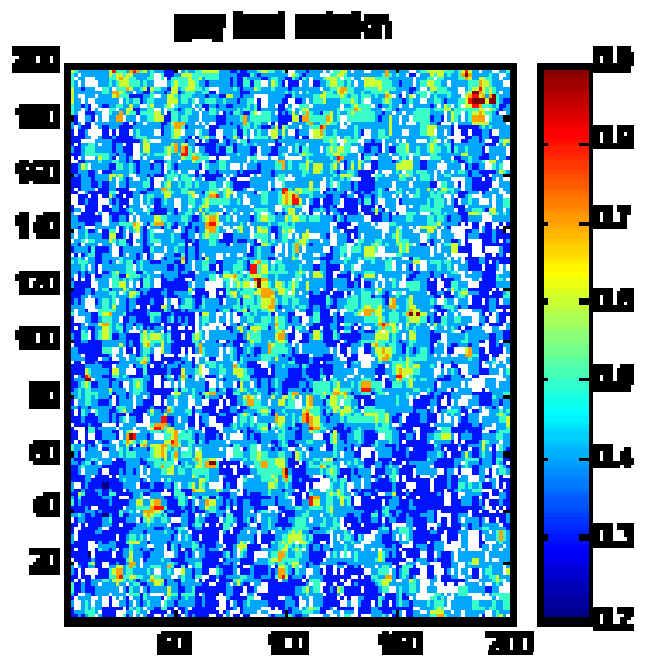

b

Fig. 1 Raw image (a) and mean grey levels in quadrats (b) 
The spun bonded nonwoven image (see fig. 1a) was used for uniformity evaluation. The starting quadrat size $2 \times 2$ pixels was selected. This size was expanded by averaging. Mean grey levels in quadrats of starting size is shown on the fig. $1 \mathrm{~b}$. These data were used for characterization of uniformity. The influence of quadrat size (expanded starting size) on the corresponding areal $\mathrm{CV}$ was investigated by using of program NONWCV.

\section{Results and discussion}

The results are part of outputs from program NONWP. The dependence of CV on the quadrat area size (program NONWCV) is given on the fig 2

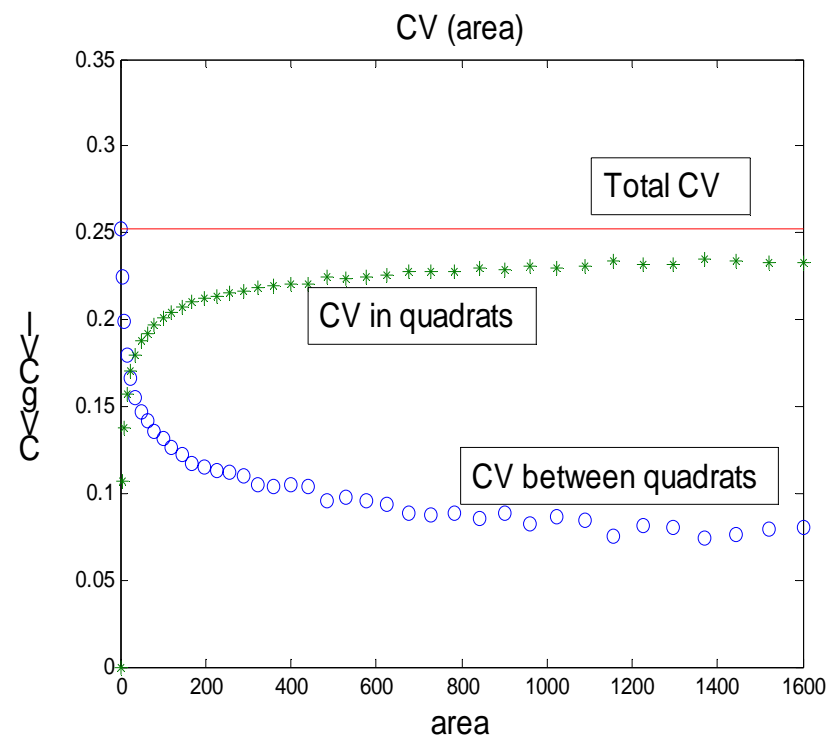

Fig. 2 Dependence of CV on quadrat size
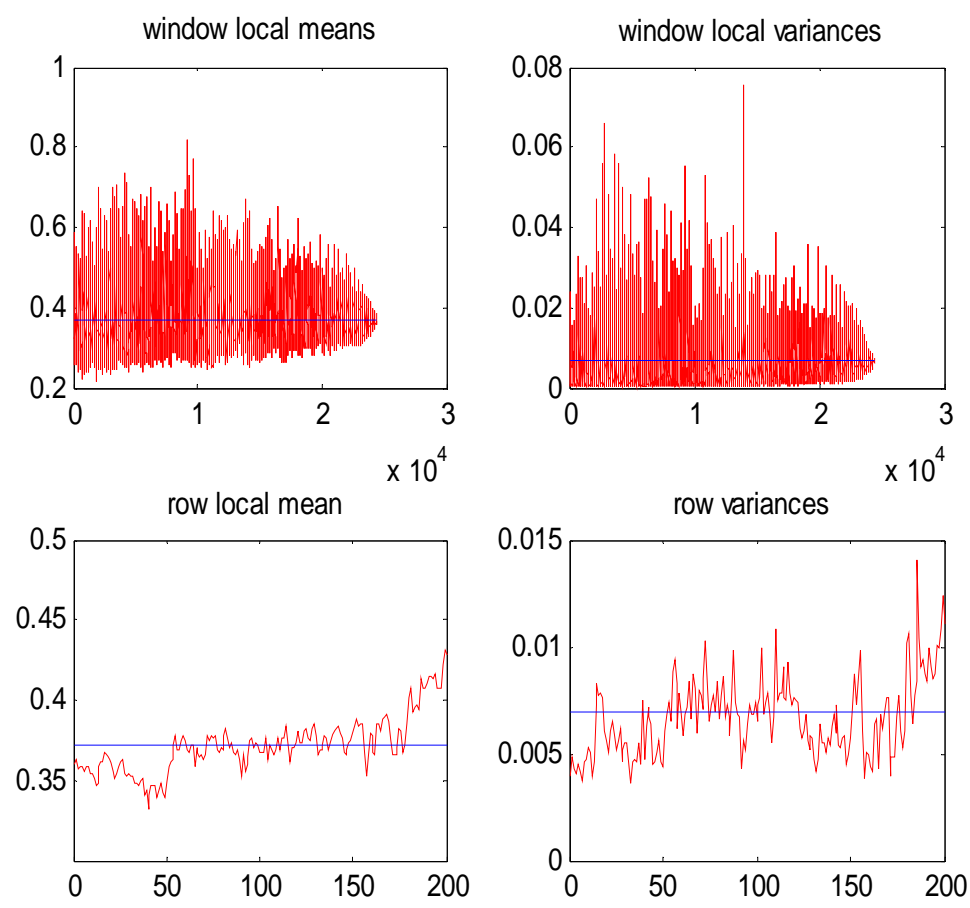

Fig. 3 Local statistics characterizing stability of mean and variance 
For deeper investigation of non uniformity the moving windows were used. Principle is division the study area to the several local neighborhoods of equal size (moving windows) and within each local window the mean and variance are computed. The dimension of moving windows can be gradually changed to obtain good identification of local anomalies. The plot of local means and variances are given in fig. 3 . The row mean and variances are shown as well.

There are visible some departures from constancy of mean and variance (stationarity). Deeper analysis of local anomalies is based on the investigation of residuals. Simple parametric model is based on the ANOVA model without interaction $z_{i j}=\mu+\alpha_{i}+\beta_{j}+\varepsilon_{i j}$. The residuals and squared residuals for this model are on the fig. 4 . The residuals were computed from total mean $\mathrm{m}$, row means $m_{i o}$ and column means $m_{o j}$ or by replacing of means by medians.
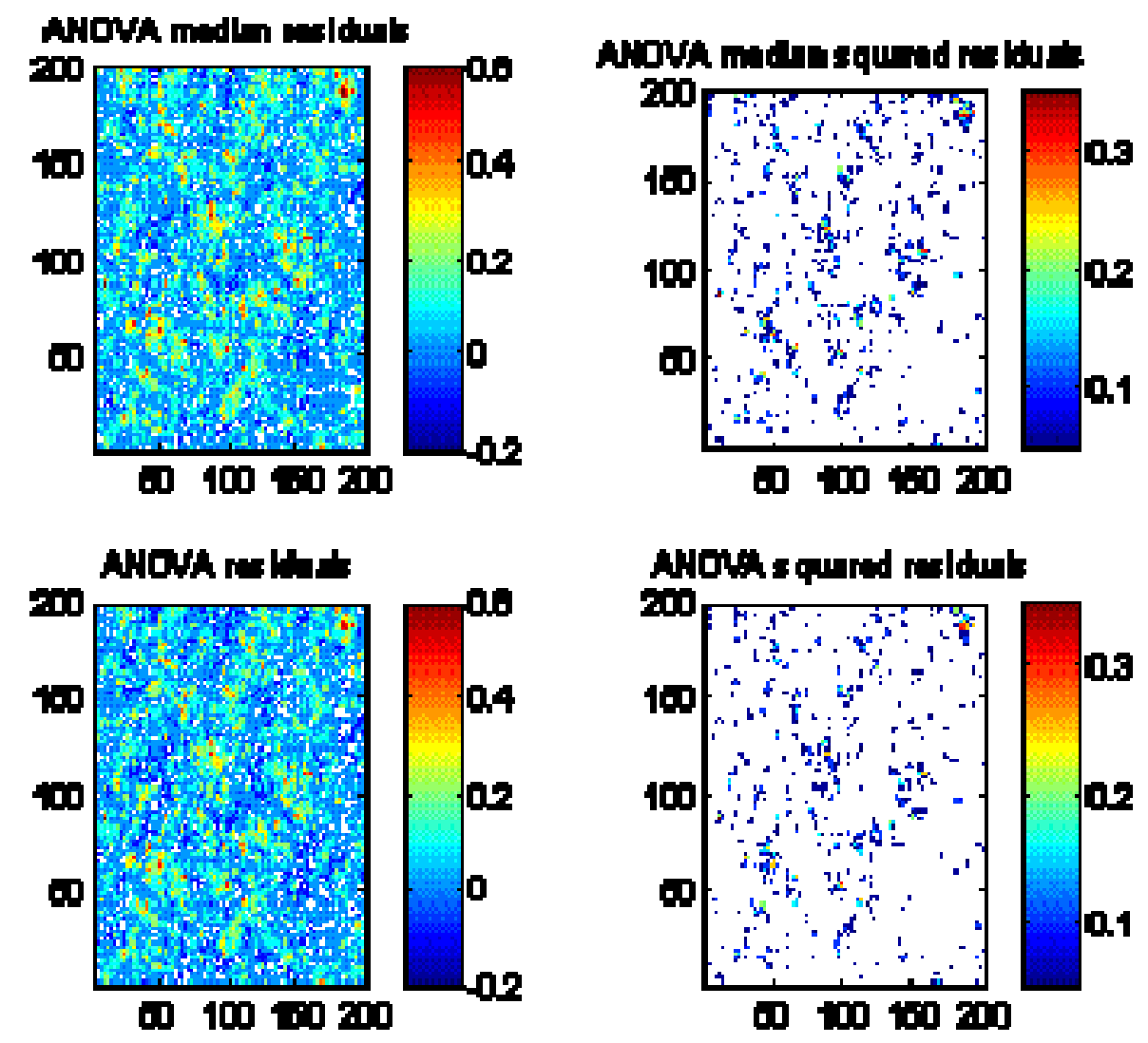

Fig. 4 Residuals and squared residuals for ANOVA model

The local "hot spots" (anomalies) are here clearly visible.

The division of total variance and index of dispersion can characterize uniformity. The division of total variance is given in the table 1.

Table 1 Variances in main directions

\begin{tabular}{llll}
\hline $\mathrm{S}_{\mathrm{L}}^{2}$ (machine) & $\mathrm{S}_{\mathrm{H}}{ }_{\text {(cross })}$ & $\mathrm{S}_{\mathrm{L}}^{2}$ (longitud.) & $\mathrm{S}_{\mathrm{T}}^{2}$ (transvers.) \\
\hline 0.0142 & 0.0176 & 0.0816 & 0.0823 \\
\hline
\end{tabular}

The $I_{d}=0.018$ is lower than limit for randomness $\mathrm{M}_{\mathrm{L}}=0.81$. ANOVA analysis leads to results that variability in both directions is not significantly different.

The variogram is machine direction, cross direction; diagonal direction and omni-variogram are shown on the fig. 5 in the $\log / \log$ form 

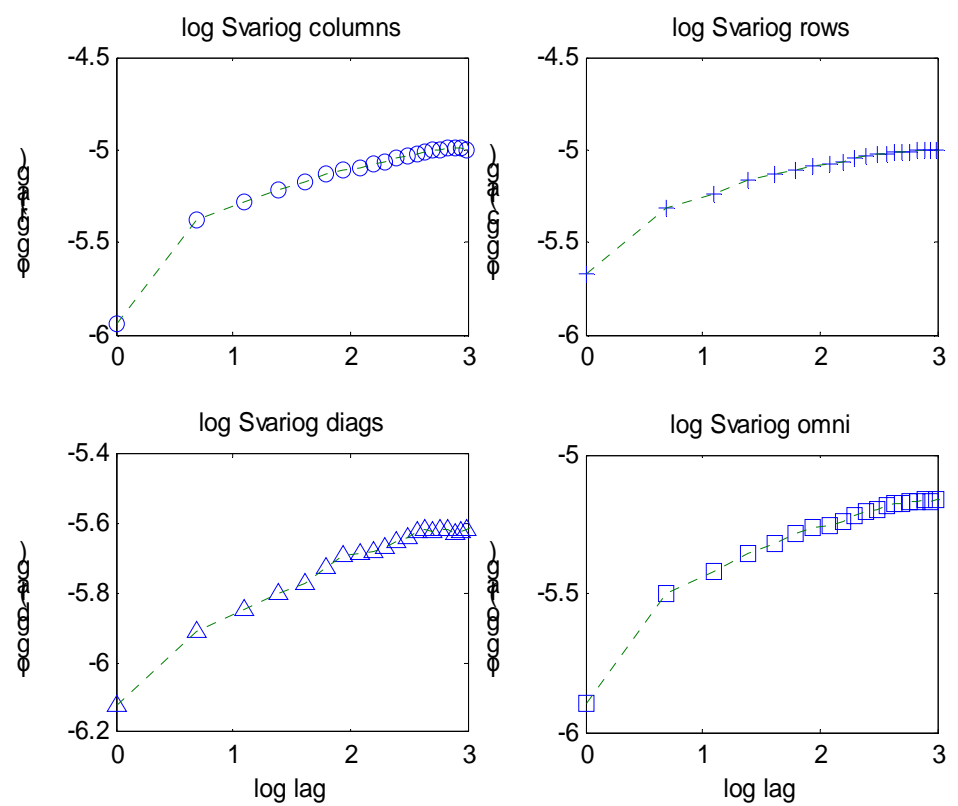

Fig. 5 Variograms in double logarithmic plot

The approximate linearity in double log plot enables calculation of fractal dimension from straight line slope [4]. The least squares estimates from initial points are: $\mathrm{D}$ rows $=2.24, \mathrm{D}$ cols $=2.16$,

$\mathrm{D}$ diag $=2.29, \mathrm{D}$ omni $=2.22$. The surface is therefore only slightly complex. The spherical model for omnivariogram (see eqn. (7)) is shown on the fig. 6.

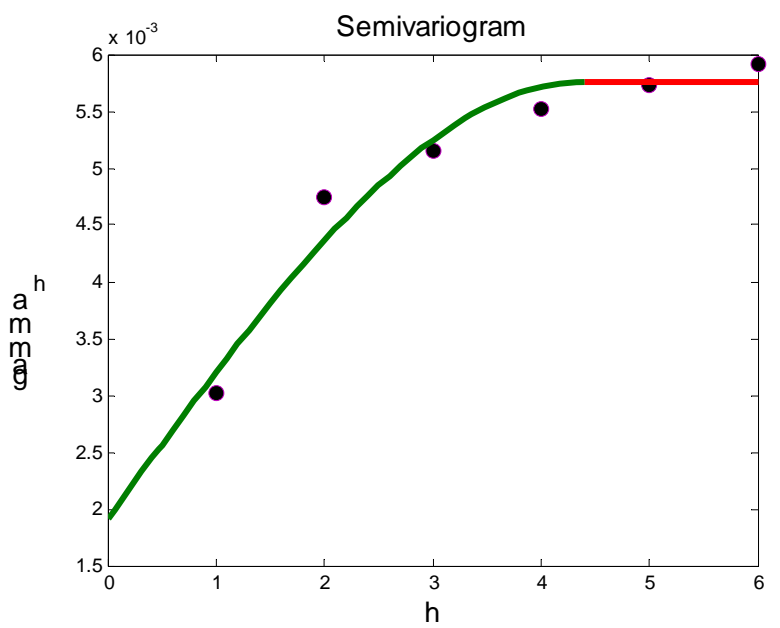

Fig. 6. Spherical model for omnivariogram

By using of nonlinear least squares the following results were obtained: Co (Nugget) $=0.019, \quad C+C o$ $($ Sill $)=0.0058$ and $a=4.402$. Due to high nugget effect the stationarity of data cannot be accepted.

\section{Conclusion}

The system of data analysis based on the above mentioned methods can be used for identification of spatial dependence for regular lattice data or planar unevenness evaluation. Tested nonwoven exhibits large-scale variation and slight complexity.

\section{References}

[1] A.D. Cliff and J.K. Ord. Spatial autocorrelation. London: Pion. 1973.

[2] N. A. C. Cressie. Statistics for Spatial Data. New York: J. Wiley. 1993. 
[3] C.W, Erickson. and J.F. Baxter. Spun bonded nonwoven fabrics. Tex. Res. J. 1973, 43: 371-378.

[4] S. Davies. Fractal analysis of surface roughness by using of spatial data. J.R. Stat. Soc. 1999, 61: 3-37.

[5] X. Huang and R.R. Bresee. Characterisation of nonwoven web structure. INDA J. Nonwovens Res. 1993, 3: $28-38$.

[6] R.Chhabra. Nonwoven uniformity. Int. Nonwoven J. 2003, Spring: 43-50.

[7] A. Cherkassky. Nonwoven uniformity characterization. Text. Res. J. 1988, 68: 242-246.

[8] J.O. Johansson. Measuring homogeneity of planar point patterns by using kurtosis, Pattern Recognition Letter. 2000, 21: 1149-1156.

[9] V.Klička. Nonwoven uniformity indices. Dissertation. TU Liberec, 1998.

[10] M. Meloun, J. Militký and M. Forina. Chemometrics for Analytical Chemistry. London: Ellis Horwood. 1992.

[11] J. Militký. Probability model of nonwovens. STRUTEX'98. Liberec, October, 1-9, 1998.

[12] J. Ord and A. Getis. Local Spatial autocorrelation statistics. Geogr. Anal. 1995, 27: 287-296.

[13] Y. Pannatier. Variowin Software for Spatial Data Analysis. New York: Springer. 1996. 